\title{
PASTA, UN ARTE CON MUCHA CIENCIA
}

\author{
Andrea Ainsa ${ }^{\mathrm{a} 1}$, Adrián Honrado ${ }^{\mathrm{a} 2}$, Pedro Marquina ${ }^{\mathrm{a}}{ }^{3}$, J. Antonio Beltrán ${ }^{\mathrm{a} 4}$, \\ Juan Calanchea a5 \\ aAgroalimentario de Aragón IA2. Universidad de Zaragoza, España. \\ a1 andreaainsa6@gmail.com, ${ }^{22}$ adrihonfri@gmail.com, ${ }^{a 3}$ pmarquin@gmail.com, a4jbeltran@unizar.es, \\ a5calanche@unizar.es
}

\begin{abstract}
Pasta is one of the most popular dishes in the world made with durum wheat as its history and regulation show. Its consumption became widespread in the 15th century, but it was in the 16th century when pasta became a frequent meal among people with few resources due to its versatility and economy. This has been recorded in books, paintings, and advertising posters from different eras. Pasta as food is so iconic that a museum based in Rome, Italy, was dedicated to it in 1993. Its history reveals fusion and eclecticism that has promoted an interesting progression from the point of view of culinary creation, but also a technological and gastronomic perspective, by increasing the existent composition and offer. From the 15th century to the present, pasta has gone from being basic sustenance to a more complex and select food, but thanks to its excellent sensory acceptance, it has never lost its popular character, nor its affordability. Therefore, faced with such a noble and recognized food, there is nothing left but to try to enrich it and continue its legacy by incorporating other ingredients such as fish or seaweed, to continue promoting its enriching transculturation.
\end{abstract}

Keywords: pasta, wheat, history, creation, art, nutrition, gastronomy.

Resumen: La pasta es uno de los platos más populares del mundo hecho con trigo duro como lo muestra su historia y regulación. Su consumo se generalizó en el siglo XV, pero fue en el siglo XVI cuando se hizo una comida frecuente entre la gente de pocos recursos debido a su versatilidad y economía. Esto ha quedado registrado en libros, pinturas y carteles publicitarios de distintas épocas. La pasta como alimento es tan emblemática que se le dedicó en 1993 la fundación de un museo con sede en Roma, Italia. Su historia revela fusión y eclecticismo que ha promovido una progresión interesante desde el punto de vista de la creación culinaria, pero también desde una perspectiva tecnológica y gastronómica, al incrementar la composición y oferta existente. Desde el siglo XV hasta la actualidad la pasta ha pasado de ser un sustento básico a un alimento más complejos y selecto, pero aun así y gracias a su excelente aceptación sensorial nunca ha perdido su carácter popular, ni su asequibilidad. Por eso, frente a tan noble y reconocido alimento no queda sino tratar de enriquecerlo y continuar su legado incorporando otros ingredientes como pescado o algas, para seguir promoviendo su enriquecedora transculturación.

Palabras clave: pasta, trigo, historia, creación, arte, nutrición, gastronomía.

Citar como: Ainsa, A., Honrado, A., Marquina, P., Beltrán, J.A., Calanchea, J. (2022). "Pasta, un arte con mucha ciencia". En: Actas del III Congreso Internacional sobre Patrimonio Alimentario y Museos. 25-26 noviembre, 2021, Valencia, España. pp. 247-263. https://doi.org/10.4995/EGEM2021.2021.13365 


\section{Introducción}

La museología es considerada por muchos como una disciplina científica independiente, cuyo objeto de estudio es la actitud específica del hombre frente a la realidad, para otros es una ciencia social surgida de disciplinas científicas documentales y contribuye a la comprensión del hombre en la sociedad (Stránsky, 1980). Por su parte, el patrimonio, se puede comprender como el "conjunto de bienes culturales y naturales, tangibles e intangibles, generados localmente, y que una generación hereda/transmite a la siguiente con el propósito de preservar, continuar y acrecentar dicha herencia" (De Carli, 2006). Desde la museología se entiende lo patrimonial como una cualidad de un patrimonio inmaterial o material que se determina por poseer tres características inseparables, que deben ser identificadas por el grupo en cuestión: pertenencia, identidad y herencia (Garma y Sansoni, 2002).

Con base en lo anterior, el patrimonio alimentario se convierte en algo esencial para estudiar, analizar y comprender, pues juega un importante papel en la configuración de la cultura de los pueblos, y de la forma cómo estas actúan y han actuado entre sí, a lo largo del tiempo. Alimentarse es tan esencial para el ser humano como el acto de respirar, formando parte de la existencia misma del hombre. Los alimentos han sido determinantes en la evolución de la raza humana; llevándonos de ser cazadores y/o recolectores hasta agricultores y explotadores de recursos naturales (Calanche, 2018). Comer es necesidad, pero también es bienestar y placer. Por eso la comida está vinculada de forma indisoluble a la cultura y el folklore de los pueblos; siendo sus alimentos factores determinantes ya que están condicionados a la disponibilidad de recursos existentes en el ámbito circundante de las comunidades. No obstante, la alimentación como patrimonio y acervo trasciende en el tiempo y es capaz de evolucionar. Gracias a las transculturaciones, fusiones y evoluciones en los ámbitos tecnológicos, gastronómicos y culinarios (Calanche, 2018). Dentro de esta perspectiva el presente estudio pretende abordar un tema curioso, interesante y digno de estudio acerca del nacimiento y evolución de un alimento emblemático, la pasta.

La historia de la pasta muestra su comienzo tras el aprendizaje por parte del hombre del cultivo de cereales, así como de su posterior molienda. Debido en gran parte a las buenas condiciones climatológicas de diferentes lugares, se incrementó la siembra y producción de pastos, entre ellos de un cereal en específico, el trigo. Este cereal era manipulado con el fin de conseguir una harina, la cual se mezclaba con agua para obtener una masa que se secaba al sol con el objetivo de mejorar su conservación, pero a su vez, también su forma de transporte. Es así como nacieron nuevos alimentos hasta entonces desconocidos como lo son hoy en día el pan, la cerveza y la pasta (Giménez, 1961).

En el siglo XV, la pasta únicamente se consumía de forma ocasional en la cocina italiana, lo que se debía a lo rudimentario de su proceso de fabricación con apenas la utilización de un molino clásico con el que molturar el trigo. Seguido a esto, se procedía a un amasado manual en el que se le daba el formato que por aquel entonces resultaba más sencillo, el espagueti, debido a su facilidad para realizarlo manualmente. Por último, se realizaba un secado durante un largo periodo de tiempo colgando la pasta, aquellas largas hebras de masa, en varillas a la sombra. Aunque este proceso ha ido evolucionando a lo largo del tiempo, la materia prima con la que se realiza este tradicional alimento y a la vez, tan especial plato, siempre ha sido el trigo duro (Triticum durum) debido a su alto contenido en proteína lo que le atribuye una gran bondad desde el punto de vista nutritivo. 
La pasta de precaria preparación en la edad media se cocinaba sola, o con azúcar y especias, aunque muchas veces estos últimos ingredientes se les incorporaba por recomendaciones médicas. Estas modificaciones (recetas) con el paso del tiempo fueron dando paso hasta las recetas que hoy se conocen y degustan habitualmente en todo el mundo. Italia fue un punto de inflexión para el cambio de percepción de la pasta; ya que durante el renacimiento en el año 1421 Bartolomé Platina, mejor conocido como de Sacchi, revolucionó la cocina y en especial el modo de ver, hacer y comer la pasta. Así finalmente pasó a la posteridad como el creador de la cocina italiana e impulsor de la actual y reconocida gastronomía italiana. A partir de este momento, ya la forma común de consumir pasta con la mano fue sustituida gradualmente por el tenedor de cuatro puntas de invención reciente para la época (Guerrero, 2019).

El consumo de pasta empezó a generalizarse y a hacerse frecuente entre la gente humilde en el siglo XVI cuando, además, se fueron ampliando los formatos utilizados. Este producto era muy asequible debido a su bajo coste y gran versatilidad. En 1700 se dio comienzo a una época de prosperidad para este tradicional alimento que hasta entonces se había obtenido en grandes superficies artesanales mezclando harina con agua y amasando con los pies como en otros alimentos (el vino), pero esto suponía una falta de higiene además de cantidades limitadas de producción. Debido a este aumento de popularidad, en el siglo XVIII se construyó una pequeña empresa pionera en la elaboración de pasta. Ya en 1823, y con años de experiencia en la elaboración de pasta, se propuso un sistema basado en el "uomo di bronzo" (hombre de bronce) un mecanismo que mejoraría este problema y facilitaría su fabricación obteniendo mayores cantidades y una mejor calidad del producto. Debido a la buena aceptación de los consumidores italianos y, por ende, mayor consumo, en el siglo XIX, se produjo una mayor industrialización. Finalmente, en 1950, este consumo se comenzó a expandir por todo el mundo para llegar al lugar que ocupa hoy en día.

La pasta no sólo ha mostrado esta evolución en su fabricación, sino que también lo ha experimentado en su forma de preparación culinaria. Ya en el siglo XV se han podido hallar diversas recetas de pasta en una antigua publicación titulada "Libro de arte coquinaria". Sin embargo, a finales del siglo XIX, la producción de pasta seca como producto industrial seguía siendo minoritaria como muestra el recetario italiano más importante del momento, es decir el Pelegrino Artusi titulado "La scienzia in cucina e l'arte de mangiare bene" de 1891 donde solo 10 recetas de las 800 que eran en total correspondían a platos de pasta seca. Durante el siglo XX, el consumo de pasta seca se extendió rápidamente en la sociedad comenzándose a "poner de moda" su elaboración tras lo cual se crearon diferentes platos como lo han demostrado numerosos cocineros y chefs tanto en libros de recetas como en sus respectivas ofertas gastronómicas.

En el caso particular de España, existen múltiples ejemplos de recetarios donde se menciona la pasta desde el siglo XVII hasta la actualidad. Ya en 1837 cuatro recetas de pasta aparecen en "El manual del cocinero" publicado por Mariano de Rementería. Aunque en años posteriores no se le presta demasiada atención a la pasta, a principios del siglo XX se inicia su consumo en otro formato, y se habla de los canelones, que durante mucho tiempo fue un plato dominical obligado de la burguesía catalana y, además, atractivo de los restaurantes más modernos de Barcelona. A su vez, también se hacen muy populares las cazuelas de fideos típicas en Cataluña, con grandes campañas publicitarias, y las "fideuas levantinas" que principalmente se preparaban con productos obtenidos de la matanza y más tarde se modernizaron con marisco como ingrediente único. Durante los años 80, una potente campaña de Pastas Gallo protagonizada por Karlos Arguiñano 
y Sofía Loren causó que este alimento pasase a un lugar privilegiado en la gastronómica ibérica y lo que se consideraba comida de pobres y de cuarteles se convirtió en un deseado plato querido por niños, deportistas y por aquellos que cuidaban su alimentación, más desde una perspectiva hedónica (Díaz, 2017). Es muy difícil que exista hoy en día, especialmente dentro del marco de la creciente globalización en que se vive, una cocina original o pura, sin influencias externas, porque éstas siempre, afortunadamente, existen para diversificar y enriquecer la dieta alimentaria (Calanche, 2009).

\section{El propósito}

En primer lugar, el objetivo de este estudio es divulgar la importancia de un alimento milenario y trascendente como lo es la pasta, desde la perspectiva de la alimentación del hombre a lo largo de su historia, con el fin de garantizar y preservar su acervo cultural, así como el rol que ha desempeñado a través del tiempo, lo que constituye para la museología, un objeto de estudio imprescindible. En segundo término, pretende identificar y valorar las propuestas existentes en la actualidad para tan noble alimento, y que constituyen para un ecléctico movimiento vanguardista la conjugación ideal entre ciencia, tecnología y arte culinario que da lugar a una excelente propuesta gastronómica.

\section{Desarrollo de la innovación}

La pasta siempre ha sido considerada un alimento básico con una gran aceptación debido a su fácil producción, transporte y almacenaje además de su versatilidad y economía. Como se ha podido ver a lo largo de su historia, la pasta ha experimentado un gran cambio tanto a nivel industrial como a nivel gastronómico. Con el paso del tiempo, la incorporación de diferentes salsas que atribuyen diferentes sabores, además, de otras matrices alimentarias que les otorga propiedades nutricionales y sensoriales a los platos elaborados con pasta ha ido cobrando importancia. Para seguir la tradición de innovar con un alimento básico y tradicional como es la pasta, se ha recurrido a la idea de incorporar nuevos ingredientes en su fabricación con el fin de conseguir mayores beneficios nutricionales con el alimento propio y no exclusivamente con la adición de salsas en sus platos. Esta nueva propuesta puede partir del uso de alimentos tradicionales, tales como: carnes, pescados, verduras, especias, huevo y/o cereales; o de otros alimentos no tradicionales, los que se denominan en inglés como novel foods -innovadores- que abarcan especialmente algas, otros cereales como la espelta, hongos e hidrolizados entre otros. Desde la linea de investigación SEA2PASTA desarrollada por el Grupo de Referencia "Calidad y Tecnología de la Carne A04_17R" de la Facultad de Veterinaria de la Universidad de Zaragoza, se pretende avanzar hacia nuevas propuestas alimenticias que integren de manera armoniosa distintos elementos de la dieta para lograr pastas de cereales con múltiples ingredientes en su composición que además han sido desarrolladas bajo la concepción de "alimento hecho a la medida" para beneficiar a la mayor cantidad de personas posibles tomando en cuenta su amplia variedad de gustos, criterios y sobre todo requerimientos (Figura 1). 


\title{
28coarta
}

\author{
Del mar a la pasta...
}

Figura 1. Logo de la linea de investigación en pasta, del Instituto Agroalimentario de Aragón (IA2).

El nuevo concepto ofrece una alternativa gastronómica que puede al mismo tiempo jugar con los sabores de la propia pasta y de las salsas que se le incorporen. Todo ello surge de una combinación de influencias del entorno, del régimen alimentario y/o del intercambio cultural. Al encontrarse España situada en una zona mediterránea, disponiendo tanto de montaña (tierra) como de océano y mares (mar), se piensa en llevar la idea de incorporar esta variada oferta de la zona, a este alimento básico y de amplia expansión a nivel mundial. Aunado a lo anterior, los tiempos han cambiado y la preocupación actual por la alimentación saludable y de calidad hace pensar en diferentes ingredientes que presenten beneficios nutricionales con la utilización de fuentes alternativas como cereales y alimentos no convencionales tanto para aquellos consumidores o clientes que lo desean como para aquellos otros que lo tengan como necesidad debido a alguna enfermedad o intolerancia alimentaria.

Como ya se ha destacado con anterioridad, las influencias culturales son muy importantes en el desarrollo de cualquier innovación y en especial cuando se trata de alimentación. La incorporación de algas para conseguir un alimento a un estilo "más oriental", de hongos foráneos para obtener un toque "más exótico" y/o de especias para obtener un producto con un sabor "peculiar" más intenso son algunas de las muestras de la transculturación en este apreciado alimento que han logrado enriquecerlo, favoreciendo su globalización. Además, es bien sabido que la pasta enriquecida con compuestos bioactivos (es decir aquellos que presentan un beneficio específico para la salud humana respaldados por la veracidad científica) y que proceden de fuentes como el pescado o las algas; tras su cocción para alcanzar el punto conocido como "al dente" antes de su ingesta representa una forma eficaz para mejorar la nutrición humana (Ainsa et al., 2021).

\section{Resultados}

La pasta actualmente es el resultado de un proceso dinámico, en permanente cambio debido a que se va ajustando a las diferentes épocas y tiempos que discurren desde su aparición. Sin duda alguna, en la actualidad, este alimento ha sido mejorado desde un punto de vista global. Se han introducido variedad de sabores combinados, logrando además incrementar su valor nutricional y su aceptación por parte de los consumidores. A día de hoy, se puede afirmar sin temor a equivocarse que se trata de un "alimento funcional" que logra el propósito establecido desde un punto de vista hedónico, gracias a la generación de particulares y acertadas sensaciones. En tal sentido, los hallazgos de esta comunicación muestran las evidencias de todo lo que ha sido, es y será este alimento. La pasta es el testimonio de una línea de tiempo admirable que necesita ser registrada, protegida, exhibida y exaltada. Todo un ejemplo, digno de admirar y para lo cual la museología se constituye en un elemento clave de entre otras muchas maneras. 
En los hallazgos, al constatar las diferentes pinturas y escritos existentes, ha quedado en evidencia el progreso de la pasta tanto en su fabricación como en su preparación, Este hecho ha representado un verdadero "salto tecnológico" donde el tiempo ha sido testigo de este vertiginoso avance, sobre todo en lo que a elaboración se refiere, pasando del más puro y rudimentario proceso manual hasta el más sofisticado mecanismo industrial. En los inicios del cultivo de trigo, las familias realizaban las labores del campo de forma manual y asimismo se realizaba el aprovechamiento de este cereal tras su cosecha, tal y como se puede observar en las pinturas de la Figura 2.

Así el proceso inicial de fabricación de la pasta se llevaba a cabo empleando un molino tradicional con una capacidad limitada de hasta 1000 kg, en el mejor de los casos, y que podían girar a razón de unas 60 vueltas por minuto. Tras la molienda se obtenía la sémola, producto burdo que posteriormente requería de un tamizado de donde se obtenía la "semolina" con la que se elaboraría la pasta. A diferencia de la cosecha y molienda, esta operación era exclusivamente realizada por mujeres y aunque todo se hacía de forma manual, ya se observaban importantes avances en el secado con el fin de facilitar su transporte. Muchas iconografías antiguas muestran este laborioso proceso (Figura 3).

Como se ha mencionado previamente en el caso específico de la pasta, la evolución en su proceso de fabricación introdujo nuevas maquinarias dentro de la que destaca el emblemático
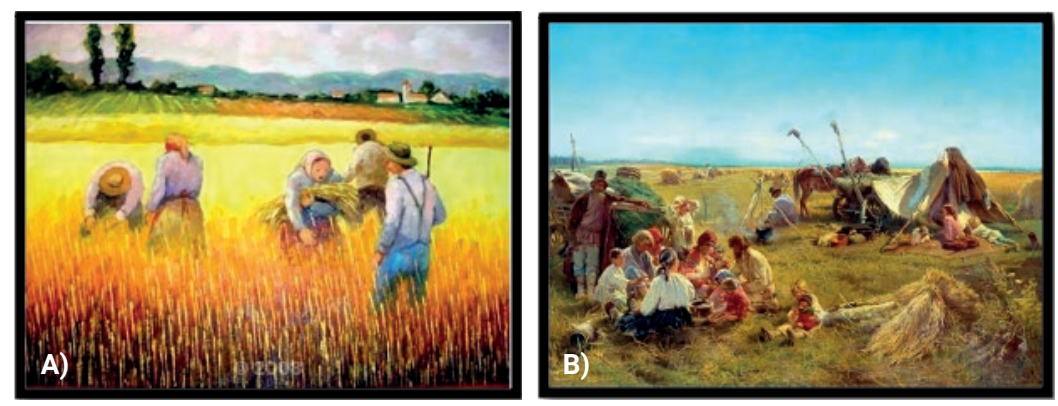

Figura 2. A) Campo de trigo de Fernando Ikoma (2008) y B) Almuerzo campesino durante la cosecha de Konstantin Makovsky (1871).
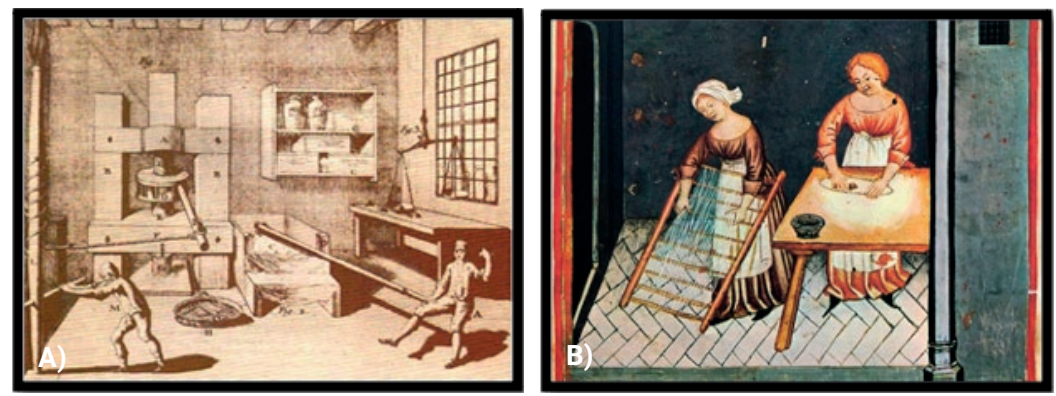

Figura 3. A) Molienda de trigo y B) Tamizado de sémola (Talla del año 1300). Fuente: https://www.baroque.it/cucina-barocca/storia-della-pasta-e-del-pomodoro.html 
"uomo di bronzo" (Figura 4). Este mecanismo pionero en su época permitió una mejora industrial de magnitud con lo cual se llegó a producir hasta $125 \mathrm{~kg}$ en 2 horas y evidentemente con una calidad mucho más homogénea. En este momento, ya se había producido una migración desde un proceso empírico, manual y limitado a las mujeres con más de arte que de ciencia, hacia un proceso mecanizado dominado por la tecnología y con la participación de hombres. Gracias al desarrollo e incorporación de máquinas y más aún después de la revolución industrial, los viejos procesos transmitidos de boca a oído y legados de una generación a otra, comienzan a coexistir con planos, formulaciones y especificaciones impresas en documentos, progresando hasta convertirse en las sofisticadas industrias que existen en la actualidad.

Este progresivo cambio en la forma de "hacer la pasta" y el aumento del "cómo comer la pasta", es decir su consumo; dio lugar a otros muchos cambios entre los que destacan los distintos formatos de presentación. En la actualidad existen un amplio número de formatos que proporcionan un gran abanico de posibilidades gastronómicas que se han ido incrementado con el tiempo. Aunque antiguamente algunos de esos formatos ya se conocían, como es el caso de la lasaña o los fideos que se elaboraban comúnmente con masa fresca, el resto de los formatos se denominaban genéricamente como macarrones.

De hecho, el término maccaronaro se refería no sólo al que vendía la pasta ya cocida, sino que también al que la producía. Se dividieron en dos categorías: el que tenían una ubicación fija y los vendedores ambulantes. Los primeros la cocinaban en su propio kiosco, en una olla grande, los macarrones venían servidos todavía calientes. Ya que aún no existía el tomate, los únicos condimentos eran aceite, sal, pimienta y los muchos quesos que se rallaban sobre los macarrones regalándole su sabor único e inimitable. Los segundos, en cambio, atravesaban la ciudad con una cesta en el cuello en el cual se transportaban los macarrones cocidos. A pesar de no ser siempre calientes consiguieron gran éxito ya que los "maccheroni" podían alimentar a toda la familia por pocas monedas (Makkeroni, 2017). El término pasta no se utilizó con el significado que se le da hoy en día, nada menos que hasta el siglo XX. Hasta entonces hablar de pasta habría significado referirse a una masa genérica para pizzas, tartas o preparaciones parecidas, así mismo los distintos formatos de pasta no se percibían como diferentes formas del mismo producto gastronómico, así, los maccheroni eran maccheroni y los vermicelli eran vermicelli, sin

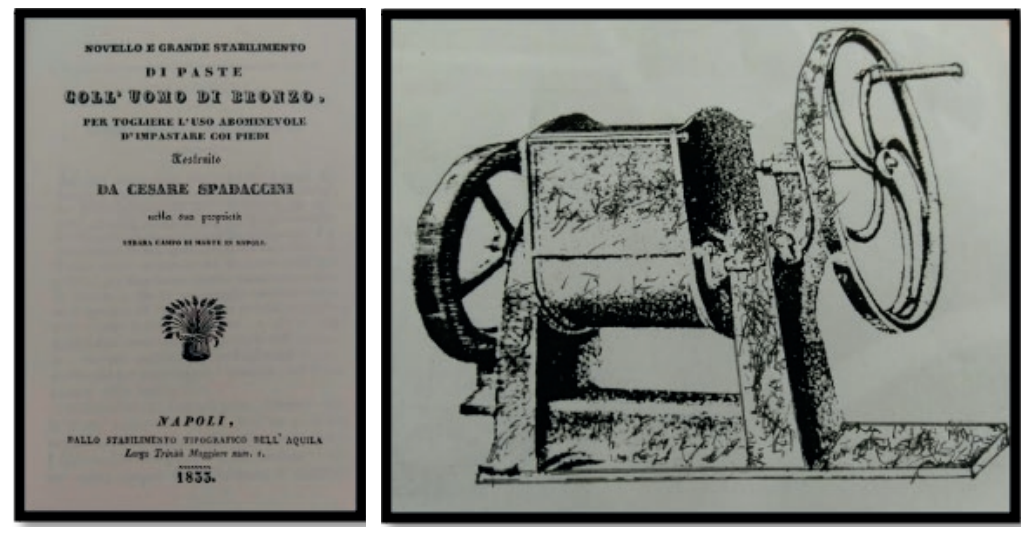

Figura 4. "il uomo di bronzo" iconografía antigua de elaboración de pasta. Fuente: http://www.medagliani.com/luomo-di-bronzo/ 
más (Coronna, 2016). En 1824 se menciona por primera vez la palabra spaguetti en la ópera titulada "Los macarrones de Nápoles" para dar nombre a la pasta seca con forma de "cordón" que se elaboraba en la época y que se comía con la mano como muestra la pintura de la Figura 5.

La costumbre de comer con la mano refleja otro interesante hecho cultural asociado a las buenas prácticas higiénicas durante la preparación y consumo de alimentos en épocas pasadas. La introducción de cubiertos y especialmente de tenedor y cucharas con el protocolo que "sentarse a la mesa” implica, conllevó a una transición positiva desde el punto de vista de la higiene personal y la sanidad pública.

Por otro lado, en cuanto a la pasta, se comienza a escribir y llevar registros de recetas y procedimientos específicos para elaborarla, especialmente los tradicionales macarrones. La figura 6 muestra la primera receta documentada de este tipo de pasta, en un libro publicado en 1390

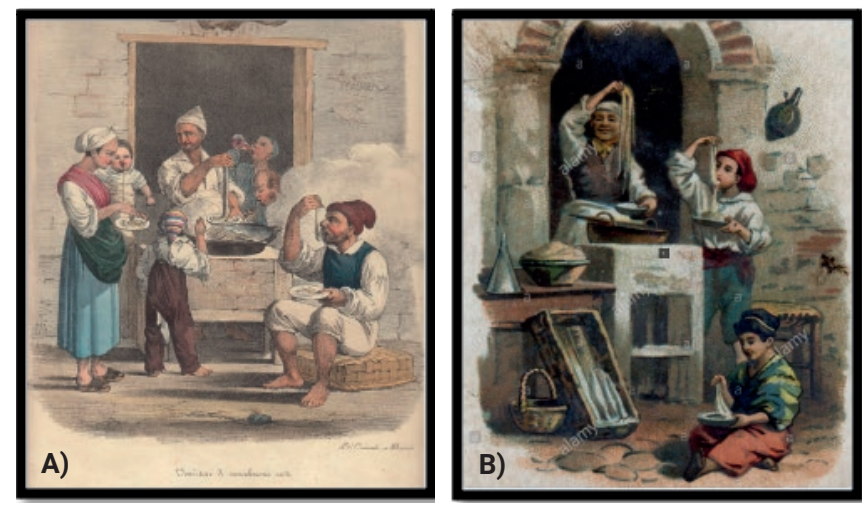

Figura 5. A) II Maccaronaro, Venditore di Spaghetti, Hjalmar Morner (1827) y B) Maccaronaro, un cuisinier preparant les pates macaroni (maccheroni) dans les rues de Nápoles. 1845. Anonimus (www.alamy.com).
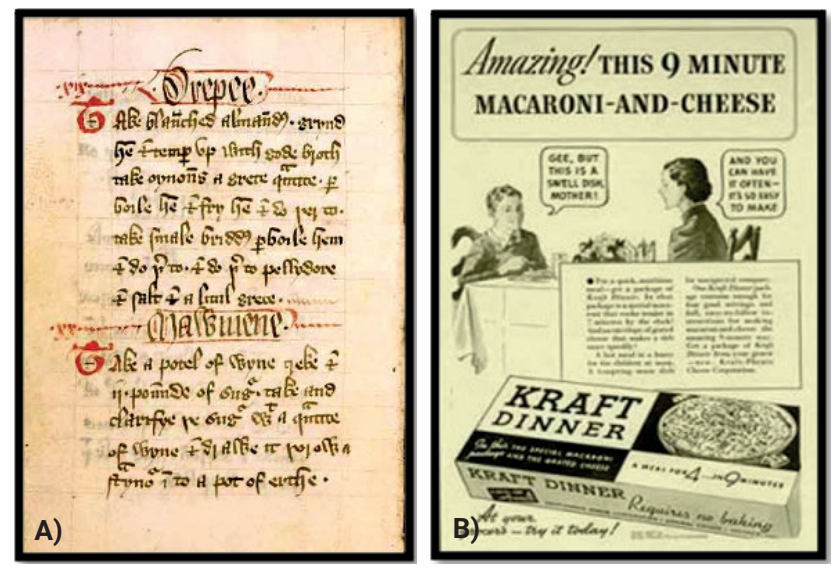

Figura 6. A) Receta original para Makerouns (1390) y B) Macarrones precocinados (1930). Fuente: Quesada (2016). 
de título "Forme of cury" que recopila las recetas elaboradas por los cocineros del rey Ricardo II de Inglaterra (A). La segunda imagen (B) por su parte, corresponde a una instrucción de uso del año 1930, siete siglos después, para macarrones precocinados en los Estados Unidos de América durante la gran depresión como comida barata y de fácil preparación.

El proceso de innovación de la pasta ha ido progresando y han surgido maneras de clasificación según su forma y corte tanto para pasta fresca como seca. En el siglo XXI, ya existen infinidades de pastas alimenticias, las especiales, las alargadas, cortas y por supuesto las rellenas, todas en centenares de formatos que se pueden encontrar tanto en supermercados como en restauración (Figura 7).

Como se ha dicho diversas clasificaciones han ido apareciendo a lo largo del tiempo, pero en todas ha estado recurrente la idea de la "pasta especial". Se trata o denomina como pasta especial aquella que se elabora con ingredientes adicionales al trigo que le atribuyen distintas características sensoriales (colores, sabores, olores o aspectos) o que simplemente logran una mejora nutricional. Todos ellos han sido indispensables en la cultura de la pasta, pero sin duda, la gastronomía también ha tenido un papel fundamental en todo esto. Como se podía leer en alguna publicación del siglo XV, los macarrones sicilianos podían cocinarse con caldo, leche o leche de almendras y servirse con queso rallado, azúcar y especias.

La sazón de quien preparaba el plato elaborado a base de pasta de trigo se constituía en un elemento determinante para su preferencia y aceptabilidad por parte de los miembros de la
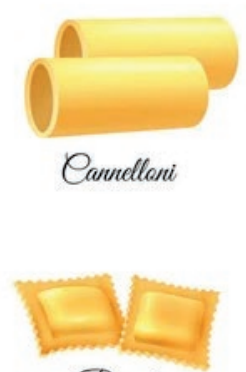

Raviali
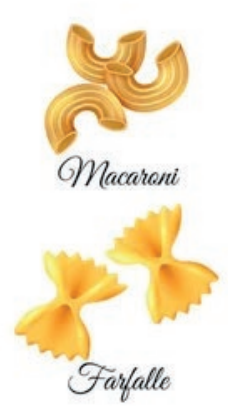

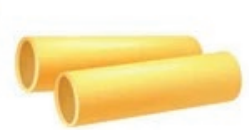

Rigateni

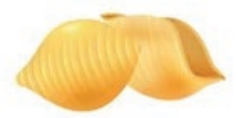

Conchigliette

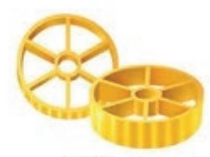

Ruate

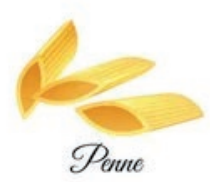

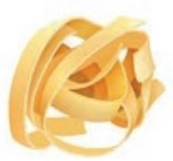

Fettucaine

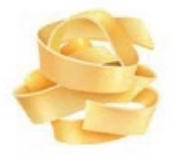

Sagliatdle

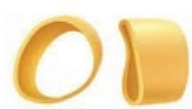

Ditaleni

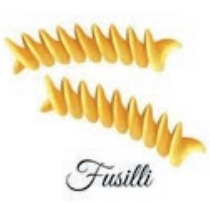

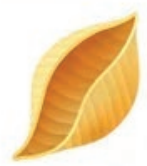

Conchiglie
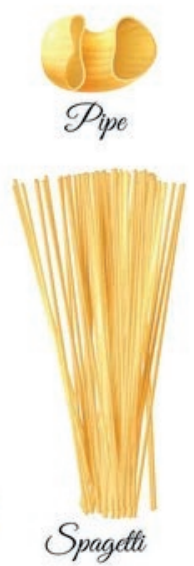

Figura 7. Formatos más comunes para pasta alimenticia. Fuente: https://es.vecteezy.com/arte-vectorial/475961-tipos-de-pasta-infografia 
sociedad. No obstante, como consecuencia de la masificación y el progreso la inclusión de salsas fue cambiando de cierto modo este toque peculiar que daban los cocineros de calle. Ahora la pasta, escurría salsa y este hecho fue el causante de la introducción del tenedor para poder comerla. El antiguo tenedor para trinchar que hasta entonces presentaba dos puntas, debido al pujante consumo de espagueti se le añadieron dos puntas más con el fin de que los susodichos se enrollasen bien. La figura 8, muestra este cambio en el hábito de consumo a través de viejas publicidades de inicios del siglo XX.

Después del siglo XX se ha producido en el caso de la pasta alimenticia una fusión extraordinaria entre tecnología, marketing y gastronomía, que va quedando reflejado a lo largo del tiempo gracias al arte, la historia y la publicidad. A continuación, y a modo de ejemplo se exponen algunos ejemplos muy ilustrativos que se han producido en España (Figura 9). En 1926, aparecía un cartel de unas pastas alimenticias al estilo italiano en el cual figuraba un cocinero comiendo espaguetis a la vieja usanza, es decir con la mano. Más tarde, este tipo de publicidad siguió vigente y en el año 1950, surgieron otros carteles como el de macarrones de la marca "Pedragosa", en el que ya se ve el sofisticado uso del tenedor de varias puntas para comer macarrones y por último el de tallarines de la marca "Castillo" donde queda claro la preferencia de los niños ante este tipo de alimento. Esto último, pasa a ser un hito para la industria de la pasta alimenticia que identifica y dirige esfuerzos hacia un público infantil ahora muy ávido de este alimento.

En todos los carteles mostrados anteriormente se trataba de pasta simple, aunque ya se incorporaban diferentes formatos por lo que el avance desde una perspectiva general era evidente. Desde entonces y hasta ahora, han surgido nuevas recetas para la fabricación de pasta, pero ahora fortaleciendo el carácter de "especial". Y así, han surgido muchas de diferentes colores con el fin de ser más atractivas visualmente y además en algunos casos pretendiendo lograr un beneficio nutricional. Algunos ejemplos de esto serían las pastas de verduras de diferentes
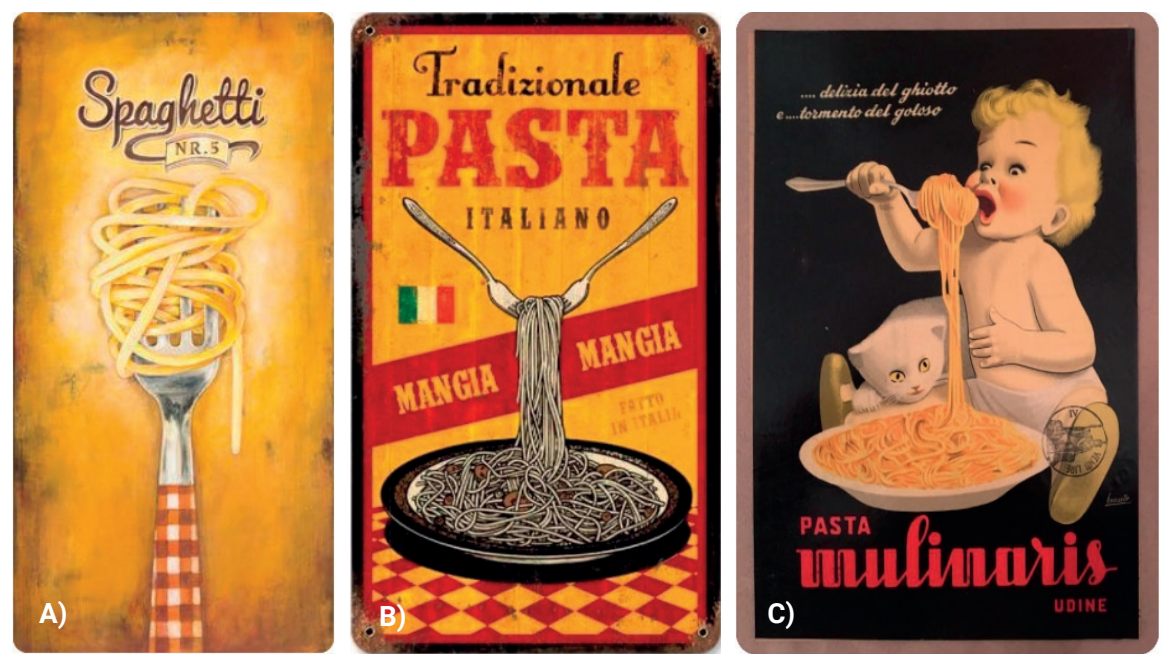

Figura 8. A) Boceto de una etiqueta, B) Publicidad comercial de una marca y C) Placa de Metal "vintage". Fuente: eu.art.com, jackandfriends.com y Old Italian food de R. Rosi. (www.pinterest.com) 
colores o la pasta con tinta de calamar para dar ese color negro que sea llamativo para el consumidor (Figura 10).

Cada día surgen nuevas gamas de pasta especiales con distintos sabores, ingredientes y propósitos un ejemplo de ello es la colección "Sabores" de la empresa aragonesa "Pastas Alimenticias Romero S.A.", con sus ofertas de pasta "con ajo y picante" y la otra al "limón y pimienta" (Figura 11).

El consumidor, ya conociendo y disfrutando de lo tradicional ahora desea que se le ofrezca algo más, sin necesidad de recurrir para esto siempre a la elaborada confección que ofrecería la restauración como opción gastronómica y por esa razón nuevos tipos de pasta, ahora con una definición que comienza a hacerse ambigua, surgen como propuestas de innovación y desarroIlo. Un ejemplo de lo anterior se tiene con el lanzamiento de los productos "la pasta del mar" que lejos de ser una linea tradicional que mantiene el trigo como ingrediente y elemento culinario
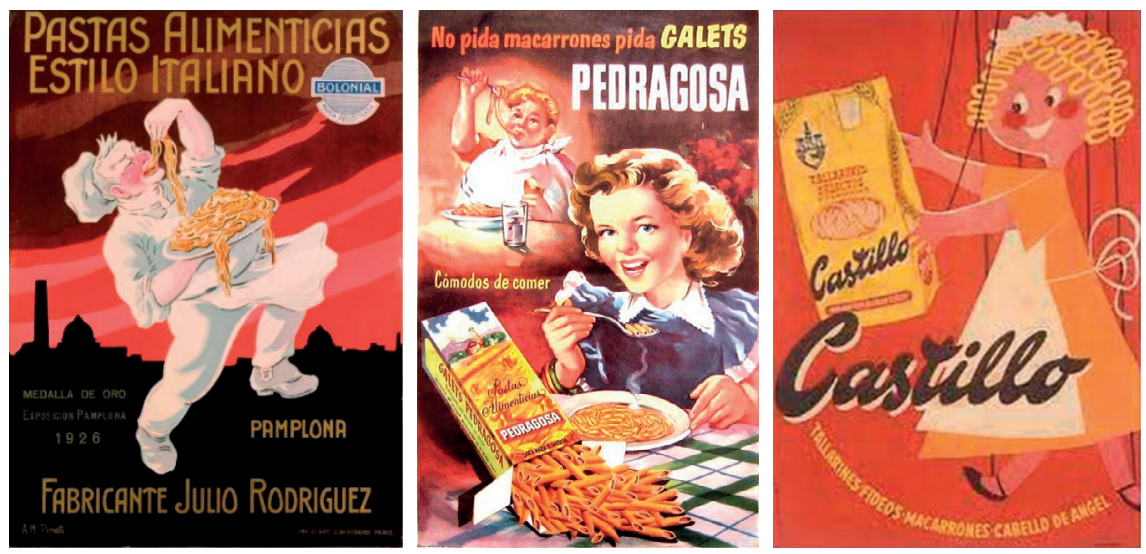

Figura 9. Anuncios comerciales de pasta alimenticia a través del tiempo. Fuente: Alimentos con Historia: Pastas alimenticias (Díaz, 2017).
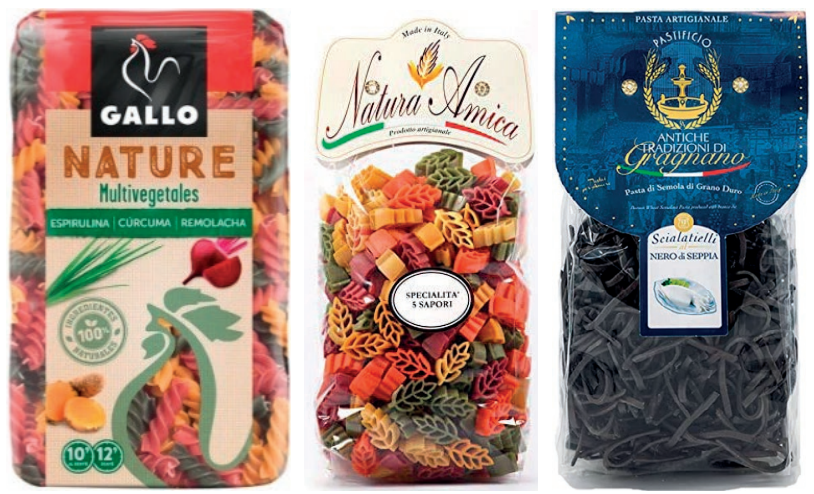

Figura 10. Pastas alimenticias del tipo especial en el siglo XXI. Fuente: www.pastasgallo.com, www.olivenoelausitalien.com y www.lb.brate.com 
principal lo sustituye por surimi de pescado extruido, a semejanza de la semolina, usando formatos corrientemente empleados por la industria de la pasta (Figura 12).

La pasta del mar constituye un ejemplo interesante, digno de quedar registrado en la historia de lo que constituye una deformación originada por una profunda influencia gastronómica de la mano de un cocinero hacia un concepto tradicional como lo es la pasta de trigo. Sin embargo, no es menos cierto que ésta surge dentro de esa corriente innovadora que busca diversificar el consumo de pescado que es un alimento de nobles bondades al igual que el trigo. Otro ejemplo, de la adaptación de la pasta a los tiempos que discurren, es la aparición de propuestas basadas en cereales distintos del trigo, con un noble propósito, hacer este tipo de alimento bien sea laminado o extruido accesible para todo tipo de consumidores incluyendo aquellos que por razones de salud (enfermedad celiaca, alergia o intolerancia) no pueden consumir trigo.

A pesar de que parezca que esta propuesta es novedosa, realmente no lo es del todo pues desde la cocina italiana siempre existieron alternativas para los productos obtenidos de la semolina, un claro ejemplo de esto lo constituían los "gnocchi" preparados a base de patatas hervidas. La figura 13 exhibe algunos de los productos que pretenden cubrir esta oferta de "pasta sin trigo".

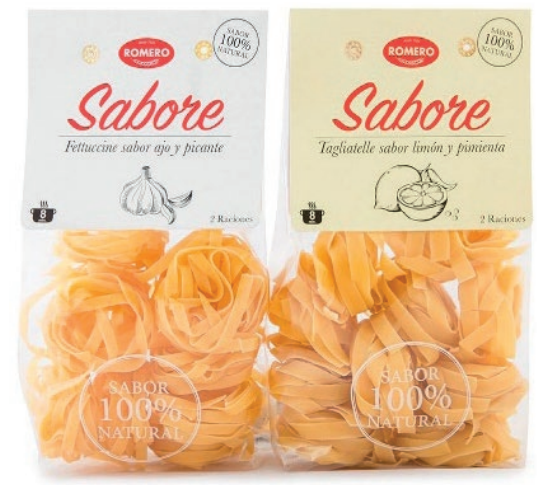

Figura 11. Pastas especiales de la marca Romero además en formato nidos que le dan un carácter gourmet. Fuente: www.pastasromero.com

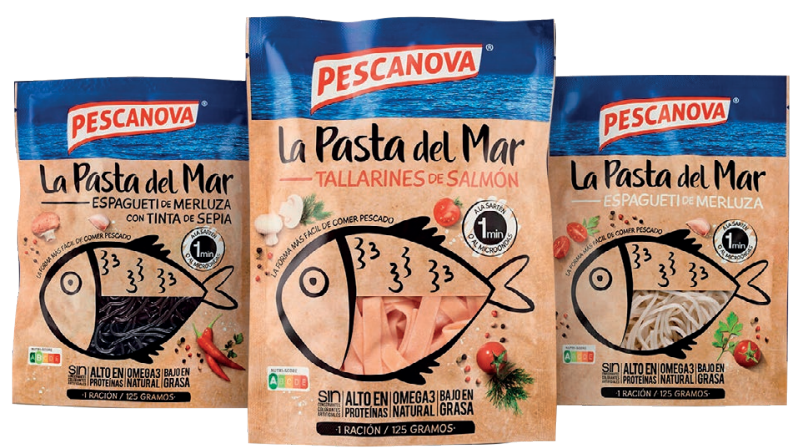

Figura 12. La Pasta del Mar, oferta gastronómica a base de pescado que emula pasta alimenticia. Fuente: www.pescanova.com 
A pesar del propósito que desee lograr la innovación introducida en el campo de la pasta alimenticia cabe destacar que desde el punto de vista de la legislación española vigente (Real Decreto 2181/1975 modificado en el 2013) todos estos innovadores alimentos de los que se han hecho referencia no son ni llegarán a ser, salvo que la norma se modifique, reconocidos como "pasta alimenticia" pues no se ha usado trigo durum en su fabricación. Por ello la propuesta de este ensayo, no busca sustituir el trigo en el diseño de los nuevos alimentos funcionales hechos a la medida, sino que apuesta por una conjugación armoniosa sensorialmente hablando, atractivo desde el punto de vista del mercado y garante del noble legado que posee el trigo como fruto de la tierra y del trabajo del hombre. Salvo aquellos casos, en que una oferta incluyente de elaboración y claro está salvando las distancias pertinentes, permita llegar a todo tipo de público. Desde el punto de vista socio-cultural e histórico la innovación pretende continuar manteniendo la esencia de la pasta como alimento derivado del trigo. Un alimento que además de aportar los nutrientes necesarios y todavía más, si se encuentra enriquecida con otros ingredientes, sea capaz de llevar a los consumidores por esas sensaciones que solo la verdadera pasta puede dar.

Antes de concluir, cabe una mención especial para el rol de la pasta dentro de la sociedad a lo largo de la historia. La pasta suele estar presentes en momentos de compartir y regocijarse en la compañía de seres queridos y como muestra de afecto. También se asocia a humildad y simpatía en personajes famosos de la historia o simplemente hace alusión a hechos sociales que marcan y han marcado la historia del hombre. En la figura 14, se observa, en primer lugar (A), la presencia de la pasta en el séptimo arte, el cine, en una película infantil inolvidable (la dama y el vagabundo, 1955, WaltDisney ${ }^{\circledR}$ ) para destacar un momento especial entre dos amantes.
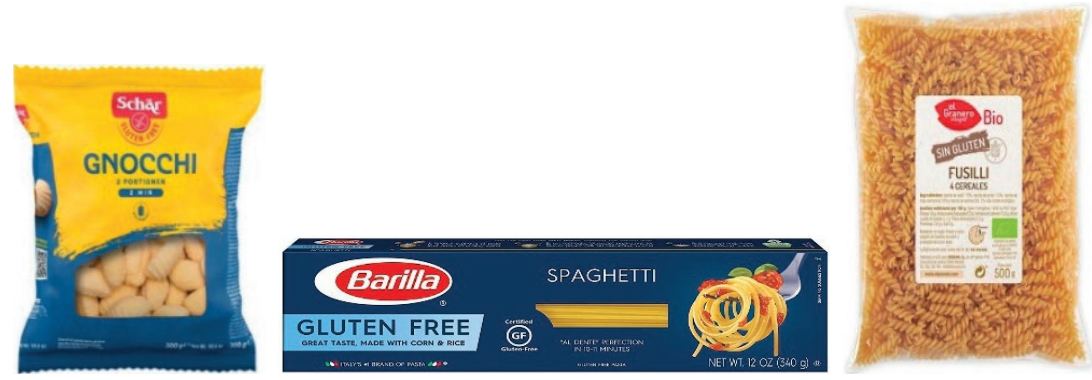

Figura 13. Pastas sin gluten: A) Ñoquis de patatas, B) Espaguetis de maíz y C) Fusillis de 4 cereales. Fuente: www.smartfooding.com, www.barilla,com y www.elgranero.com
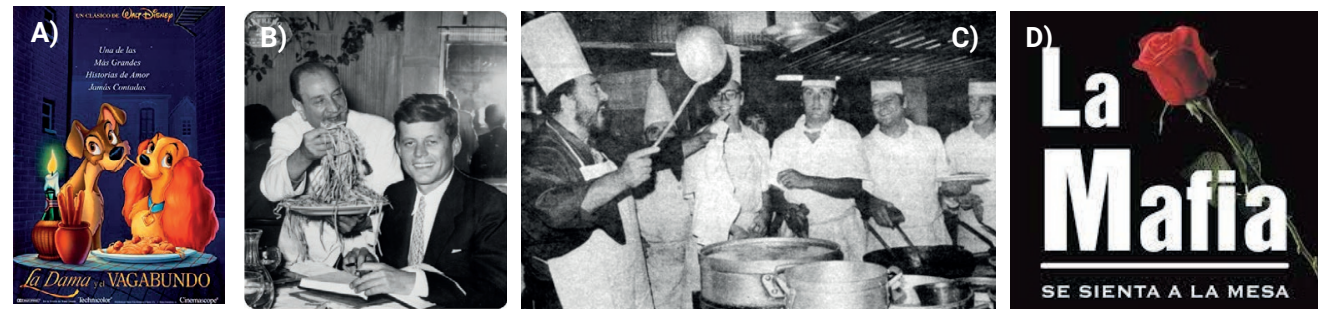

Figura 14. Imágenes diversas de personajes reales y ficticios de la historia. Fuente: Google Images (2021). 
En segundo término (B), se muestra una ilustración del icónico presidente de USA J.F. Kennedy degustando un plato de linguini Alfredo junto a su creador Alfredo Di Leio durante su visita a Roma (1957). Seguidamente se observa al famoso tenor L. Pavarotti cocinando pasta durante su visita a Bilbao en el restaurante el Bermeo en 1978. Por último, destaca el logo (2021) de una empresa con más de 20 años de trayectoria en España, dedicada a la elaboración y venta de pasta que aprovechando la tradición culinaria de una tierra (Sicilia) la vinculó a una de las más famosas organizaciones clandestinas, surgida también en su mismo seno.

\section{A manera de conclusión}

La pasta más allá de un alimento es una acervo histórico y cultural que prevalecerá en el tiempo para las generaciones futuras. La mejor forma de salvaguardar ese legado es mantener en lo posible su esencia viva y sin intromisiones. Promover su consumo y garantizar el suministro en cantidad y calidad de los ingredientes necesarios para su preparación. Tanto su fusión como su evolución gastronómica debe ser armoniosa y respetando los rasgos diferenciadores que la caracterizan. No obstante, como el producto de la mente del hombre que es no está exenta de ser modificada, pudiendo ser sometida a mejoras eclécticas mediante combinaciones tecnológicas, culinarias y artísticas que, pese a que supongan cambios en el alimento, sigan manteniendo desde un punto de vista técnico y económico, su esencia como el producto emblemático que es, ya que hoy en día resulta indispensable para las dietas de muchos seres humanos en cualquier parte del mundo como resultado de la postmodernidad que caracteriza a este siglo XXI.

\section{Referencias}

AINSA, A. IRANZO, L. HONRADO, A. MARQUINA, P. RONCALÉS, P., BELTRÁN, J. y CALANCHE, J. (2021). "Effects of cooking over the stability of fatty acids as bioactive compounds in enriched pasta with a fish by-product". En: Cereal chemistry. https://doi.org/10.1002/cche.10481

DE CARLI, G. (2006). "Los museos latinoamericanos”. En: La gaceta de museos, 37 (febrero - mayo), pp. 8-11.

CALANCHE, J. (2009). "Influencias culturales en el régimen alimentario del venezolano". En: Anales Venezolanos de Nutrición, 22(1), pp. 32-40.

CALANCHE, J. (2018). La alimentación en la sociedad venezolana: un devenir histórico. Editorial Académica Española.

CORONNA, C. (2016). L'alto forno. http://www.laltoforno.com/acerca-la-pasta-una-cuestion-identidad/ [Consulta: 15 de septiembre de 2021]

DÍAZ YUBERO, I. (2019). "Pastas alimenticias". En: Díaz Yubero, Ismael. Alimentos con historia. Madrid: Mercasa.

España. Decreto 2181/1975, de 12 de diciembre, por el que se aprueba la Reglamentación TécnicoSanitaria para la Elaboración, Circulación y Comercio de Pasas Alimenticias. BOE, 13 de septiembre de 1975, núm. 220. 
GARMA, E. y SANSONI, A. (2002). “Museo y desarrollo personal. La apropiación personal del contexto patrimonial como desafío museológico". Tesis de grado. Universidad Tecnológica Equinoccial, Quito, Ecuador.

GIMÉNEZ, O. (1961). Del trigo y su molienda. Buenos Aires: Editorial Universidad de Buenos Aires.

GUERRERO NAVARRETE, A. (2019). Diseño de tenedor para plato fuerte. Instituto tecnológico de Celaya, México.

QUESADA, F. (2016). Macarrones con queso. https://www.frquesada.com/macarrones-con-queso/ [Consultada: 2 de septiembre de 2021].

MAKKERONI. (2017). Historia de la pasta, los "maccaronari". https://makkeroni.cat/historia-de-lapasta-los-maccaronari/ [Consultada: 25 de agosto de 2021].

STRÁNSKY, Z. 1980. "Museology - Science or Just practical museum work?" En: Museological Working Papers, 1, pp. 42-44.

"La historia de la pasta italiana - documental historia - historia de la comida. La Ha de Italia" Youtube $<$ https://www.youtube.com/watch?v=D6tScU_AYp8>. 